\title{
Tratamento de Classe II, Divisão 1, com ausência congênita de incisivo lateral superior
}

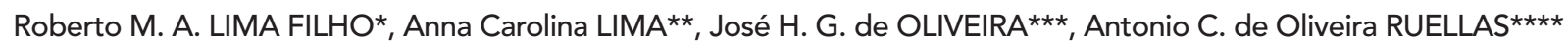

Resumo

Este relato mostra tratamento Ortodôntico efetuado em paciente portador de Classe II, Divisão 1, de Angle, com sobremordida profunda e agenesia do incisivo lateral superior esquerdo, em que o espaço foi fechado ortodonticamente e o canino ocupou o lugar do incisivo lateral. Os procedimentos adotados possibilitaram a obtenção de estética agradável e relação oclusal normal. A linha mediana não apresentou desvio ao término do tratamento e a discrepância vertical foi devidamente corrigida.

Palavras-chave: Agenesia de incisivo lateral. Classe II, Divisão 1. Estética dentária.

\section{INTRODUÇÃO}

Uma das dificuldades na Ortodontia é a correção de más oclusões com ausência congênita de incisivo lateral superior. Nesses casos, o planejamento geralmente inclui fechamento ou abertura de espaços ${ }^{2,3,5}$.

Alguns autores sugerem que a abertura de espaço para colocação de próteses convencionais ou sobre implantes, posicionando-se os caninos em relação Classe I de Angle resulta em melhor oclusão e produz menor achatamento no perfil facial ${ }^{4}$. Com o advento dos implantes ósteo-integrados, essa opção teve maior aceitação.

Em casos de fechamento de espaço, para que resultados satisfatórios sejam obtidos, os caninos devem ser alterados em seu contorno por meio de desgaste e/ou restauração. Nesses casos, a medida em que a oclusão posterior se desloca de Classe I para
Classe II, torna-se fundamental a colaboração do paciente no uso de mecânicas de protração. A escolha adequada do plano de tratamento depende do tipo de má oclusão, tamanho e estética do canino.

\section{RELATO}

Paciente MP com 9 anos, gênero masculino, foi encaminhado ao consultório para avaliação e tratamento de má oclusão. Ele era ansioso e tinha hábito de ranger os dentes como resultado de comportamento inconsciente para colocar um maior número de dentes em contato. Amígdalas e adenóides foram retiradas anteriormente. O perfil facial correspondia ao tipo convexo-mesognático. A musculatura labial era hipotônica e a altura inferior da face parecia ser menor em relação à sua altura total. O paciente apresentava agenesia do incisivo lateral superior esquerdo.

\footnotetext{
* Diplomado pelo American Board of Orthodontics.

** Mestre em Ortodontia pela Marquette University, Milwaukee, EUA.

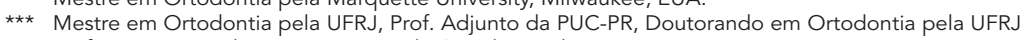

$\star \star \star \star$ Professor Doutor do Departamento de Ortodontia da UFRJ.
} 
Decidiu-se aguardar o desenvolvimento dentário para início do tratamento ortodôntico. A figura 1 mostra fotografias faciais e intra-orais do paciente aos 11 anos, quando foi realizada documentação completa. A análise cefalométrica revelou padrão Classe I esquelético. $\mathrm{O}$ ângulo do plano mandibular era reduzido $\left(16,5^{\circ}\right)$, compatível com seu padrão de crescimento, conforme telerradiografia lateral (Fig. 2A). O ângulo de convexidade de Downs foi $5^{\circ}$ (Fig. 2B). Tanto a maxila quanto a mandíbula estavam bem posicionadas em relação à base anterior do crânio (SNA $=81,6^{\circ}$ e $\left.\mathrm{SNB}=77,5^{\circ}\right)$. A queixa principal do paciente era a protrusão dos incisivos superiores e ausência do incisivo lateral.
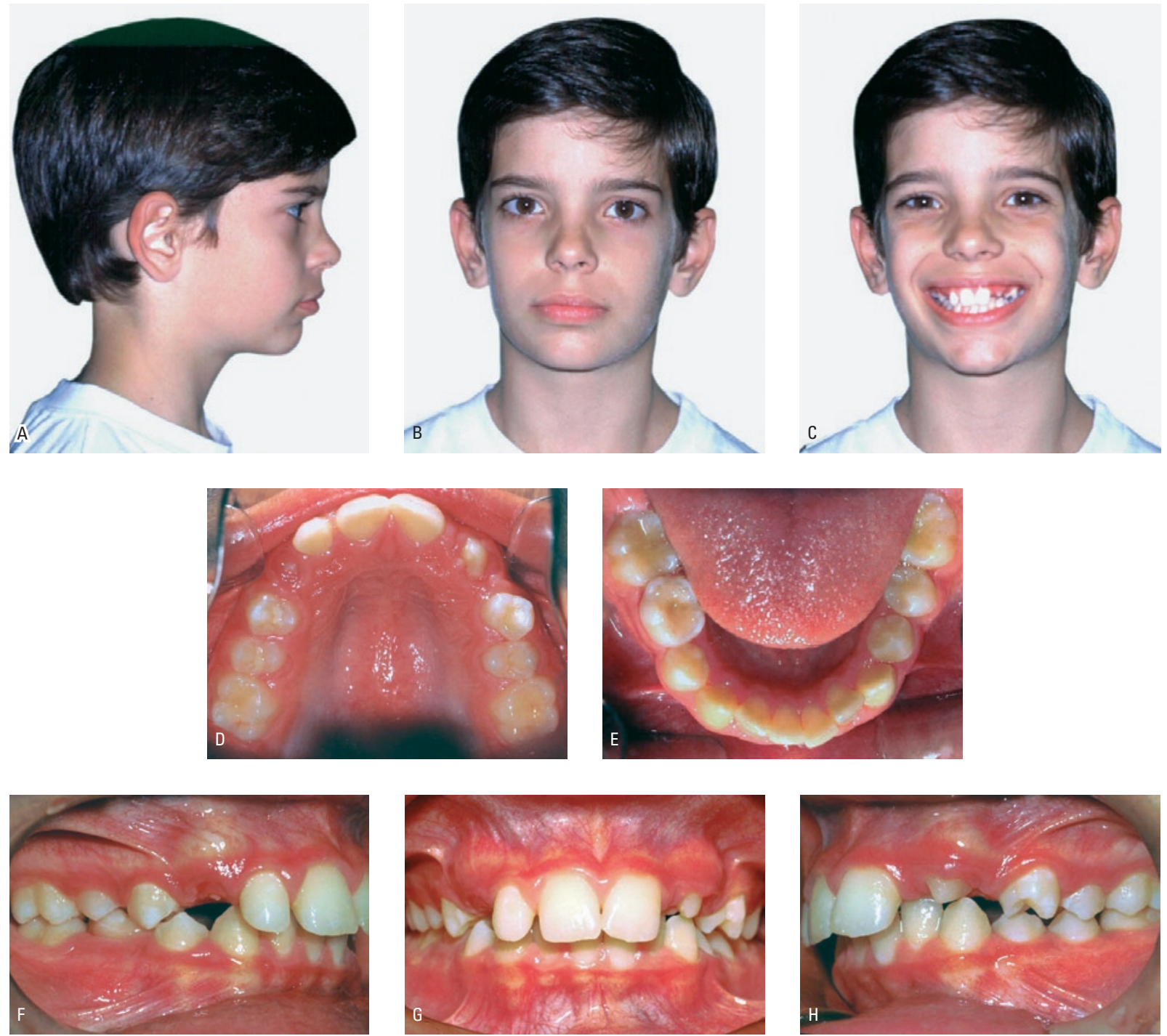

FIGURA 1 - Fotografias intra-orais e faciais do paciente MP, 11 anos, na fase pré-tratamento: A) Perfil direito; B) Frontal em repouso; C) Frontal em sorriso; D) Oclusal maxilar; E) Oclusal mandibular; F) Intra-oral direita; G) Intra-oral anterior; H) Intra-oral esquerda. 

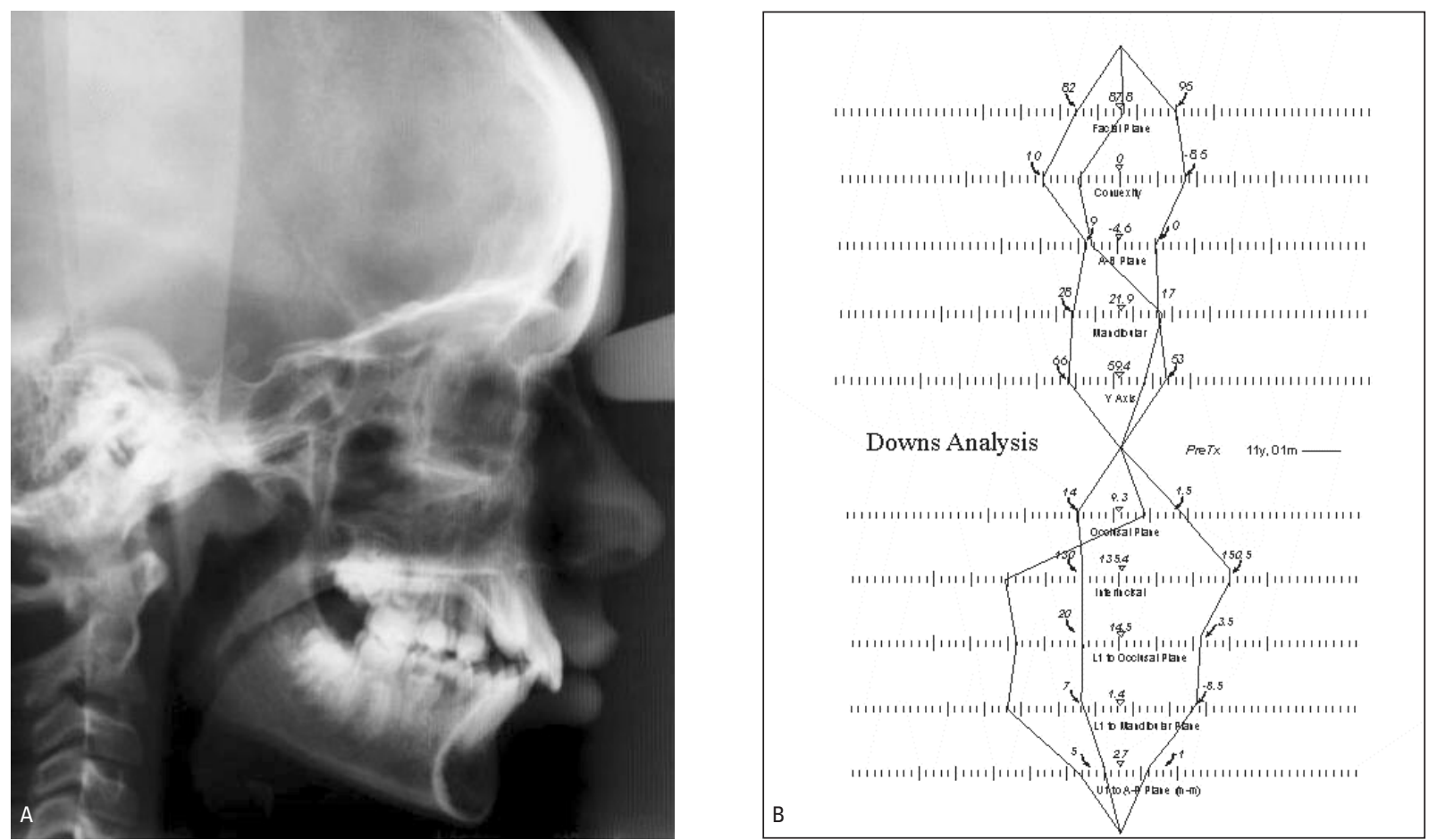

FIGURA 2 - A) Teleradiografia cefalométrica lateral do paciente MP, 11 anos e B) Análise cefalométrica de Downs.

\begin{tabular}{|c|c|}
\hline \multicolumn{2}{|r|}{ Tabela 1 - Objetivos do tratamento. } \\
\hline Local & Objetivos Específicos do Tratamento \\
\hline Maxila & Manter a dimensão transversal \\
\hline Mandíbula & Estimular rotação horária durante o tratamento \\
\hline Dentição Maxilar & $\begin{array}{l}\text { Eliminar mordida cruzada posterior; corrigir discrepância vertical; nivelar e alinhar dentes; obter relação molar } \\
\text { assimétrica; deslizar segmento posterior esquerdo para frente visando relação Classe II }\end{array}$ \\
\hline Dentição Mandibular & Nivelar e alinhar dentes; evitar movimentação anterior dos incisivos \\
\hline Oclusão & $\begin{array}{l}\text { Corrigir discrepância ântero-posterior (lado direito); relação Classe II no lado esquerdo; reduzir sobressaliência; } \\
\text { corrigir sobremordida; estabelecer boa oclusão funcional }\end{array}$ \\
\hline Estética & Melhorar características do sorriso e estética dentária \\
\hline
\end{tabular}

\section{ETIOLOGIA}

Neste caso, a etiologia poderia ser uma combinação de fatores genéticos.

\section{DIAGNÓSTICO}

Classe II, Divisão 1.

\section{LISTA DE PROBLEMAS}

- Classe II;
- Agenesia do incisivo lateral superior esquerdo;

- Sobressaliência excessiva;

- Sobremordida profunda;

- Mordida cruzada posterior;

- Apinhamento dos incisivos inferiores;

A relação dos objetivos específicos de tratamento está listada na tabela 1. 


\section{TRATAMENTO}

Decidiu-se fechar o espaço do incisivo lateral superior mantendo-se o segmento posterior esquerdo em relação Classe II. Inicialmente, foi corrigida a mordida cruzada por meio de elásticos intermaxilares antes da montagem de aparelhagem fixa. Aparelho extra-oral de tração cervical com força assimétrica e plano de mordida foram colocados. Todos os dentes foram bandados e/ou colados, sendo os arcos superior e inferior nivelados, alinhados e correlacionados. Plano de mordida foi confeccionado com molas digitais, contornando a superfície distal do primeiro molar superior esquerdo para evitar que houvesse movimentação desse dente em sentido distal pela tração cervical. Foi planejada a perda de ancoragem do segmento posterior esquerdo, possibilitando migração dos dentes anteriormente, evitando-se na medida do possível, desvio da linha mediana. Elásticos intermaxilares nos sentidos Classe II e Classe III com forças de baixa magnitude foram utilizados na correção da discrepância ântero-posterior e linha mediana.

A colaboração do paciente foi inadequada, não seguindo prescrições para uso do aparelho extraoral e não comparecendo a diversas consultas. Além disso, a higiene oral foi deficiente. O tratamento corretivo durou 34 meses.

$\mathrm{Na}$ contenção planejou-se utilizar placa Hawley maxilar e fixo mandibular 4 a 4 . Dentes anteriores superiores foram colados pela lingual com fio twist flex para manter espaços fechados.

\section{DISCUSSÃO}

Agenesias uni ou bilaterais dos incisivos laterais superiores geralmente conduzem a situações esteticamente desagradáveis. Nesses casos, existem duas alternativas para o tratamento, abertura ou fechamento de espaço.

a) Abertura de espaço para o incisivo lateral esquerdo ausente

A abertura de espaço e colocação de implantes é indicada particularmente em casos com es- paços generalizados entre os dentes, tendência à Classe III com mordida anterior topo-a-topo e agenesia concomitante de outros dentes. O local para colocação do incisivo lateral ausente seria obtido pela redistribuição dos dentes espaçados. Quando se abre espaço, a principal preocupação é a largura da borda alveolar na região do incisivo lateral ausente. Essa dimensão pode ser influenciada na dentição mista durante a erupção do canino permanente. A situação ideal seria estimular a erupção desse dente próximo ao incisivo central permanente. Após sua erupção, o canino pode ser movimentado para distal em sua posição normal. Com essa movimentação, forma-se largura vestibulolingual adequada da borda alveolar, facilitando a colocação de implante ${ }^{1}$.

As vantagens dessa opção incluem facilidade na obtenção de simetria e estética na região anterior da maxila e simplificação do tratamento. Por outro lado, as desvantagens compreendem necessidade de contenção a longo prazo com incisivo lateral provisório e colocação de implante após o término do crescimento ou desgaste dos dentes adjacentes para colocação de prótese convencional.

b) Fechamento de espaço para o incisivo lateral esquerdo ausente

Nesse caso, a transformação do canino superior esquerdo em incisivo lateral seria necessária. Dentre as desvantagens, estariam a dificuldade na correção da linha mediana, o comprometimento da estética anterior resultante da variação no tamanho e morfologia dos dentes, e dificuldade na combinação do canino esquerdo com o incisivo lateral direito.

Apesar desses fatores, estética agradável e relação oclusal excelente foram atingidas. A movimentação mesial do molar superior esquerdo e sua rotação foram efetuadas visando facilitar intercuspidação posterior. Relação molar assimétrica e boa intercuspidação foram obtidas com relação Classe II no lado esquerdo. A linha mediana não apresentou desvio ao término do tratamento e a discrepância vertical foi devidamente corrigida. 
$\mathrm{Na}$ resolução das diferenças no tamanho e morfologia dos dentes, o canino superior esquerdo foi transformado em incisivo lateral e o incisivo lateral direito foi aumentado, visando obter simetria com o canino esquerdo. As coroas clínicas do canino superior esquerdo e primeiro pré-molar foram aumentadas para melhorar a estética. A figura 3 mostra fotografias faciais e intra-orais do paciente aos 14 anos na fase pós-tratamento. A superposição total revelou que a face cresceu para baixo e para frente.
O ângulo ANB diminuiu $1,5^{\circ}$ e o ângulo do plano mandibular não apresentou alteração durante o tratamento. A figura 4 mostra telerradiografia lateral e radiografia panorâmica na fase pós-tratamento. A análise de Downs nas fases pré-tratamento, progresso e pós-tratamento encontra-se na figura 5 . Os pais e o paciente ficaram plenamente satisfeitos, pois esse caso de ausência congênita de incisivo lateral foi tratado sem a necessidade de utilização de implante ou prótese fixa.
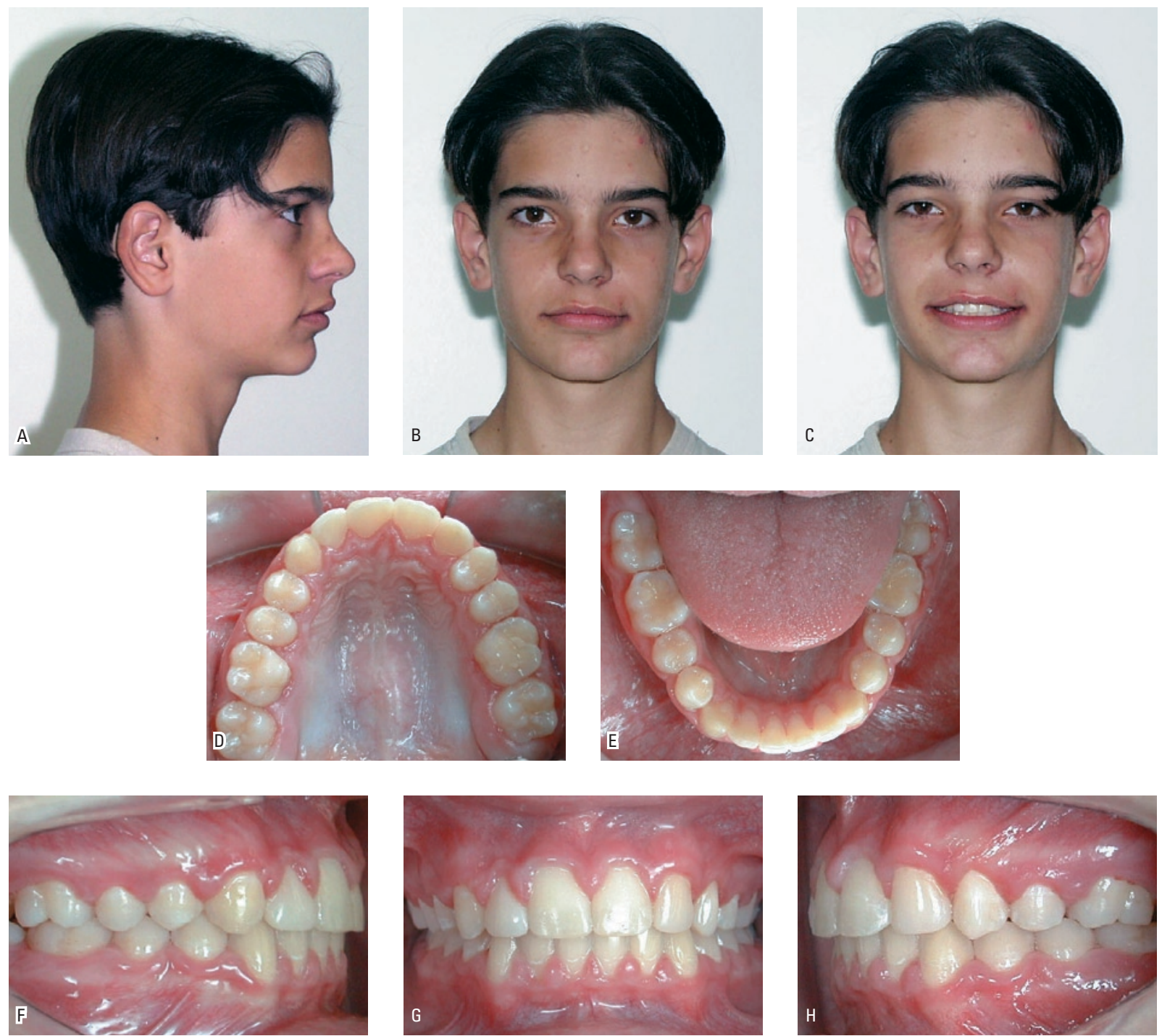

FIGURA 3 - Fotografias intra-orais e faciais do paciente MP, 14 anos, pós-tratamento: A) Perfil direito; B) Frontal em repouso; C) Frontal em sorriso; D) 0clusal maxilar; E) Oclusal mandibular; F) Intra-oral direita; G) Intra-oral anterior; H) Intra-oral esquerda. 


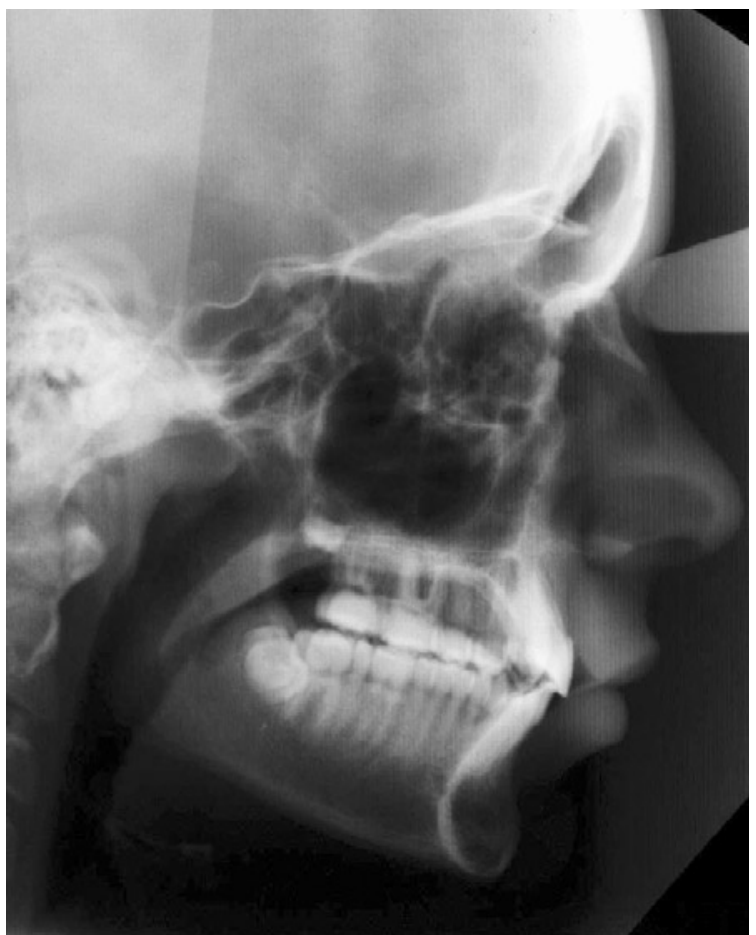

FIGURA 4A - Telerradiografia lateral do paciente MP, 14 anos após tratamento.

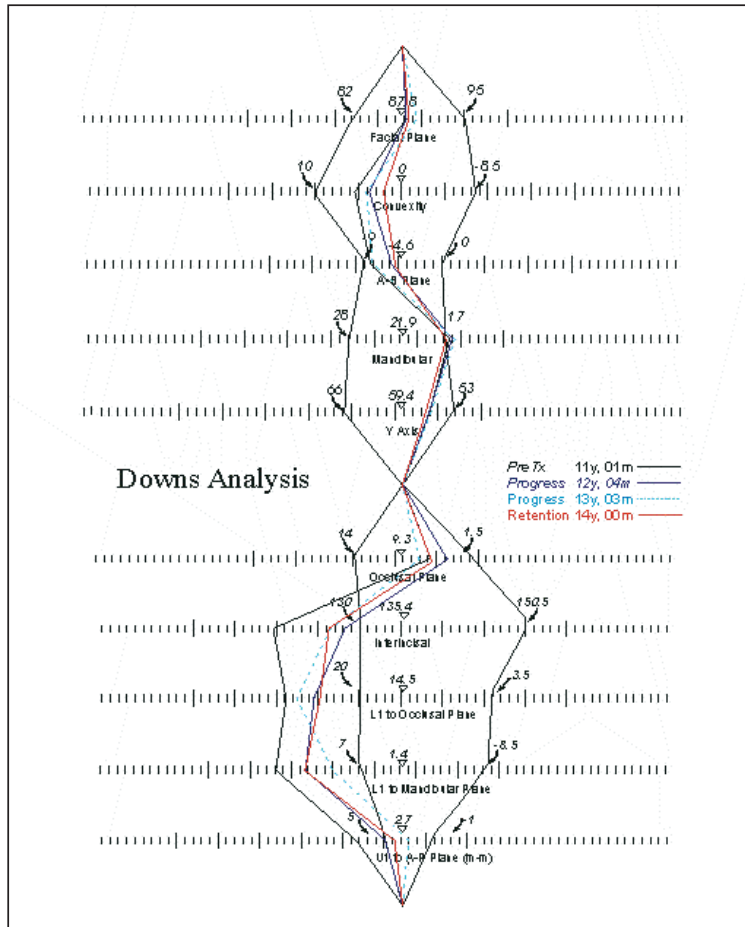

FIGURA 5 - Análise de Downs nas fases pré-tratamento, progresso 1 progresso 2 e pós-tratamento.

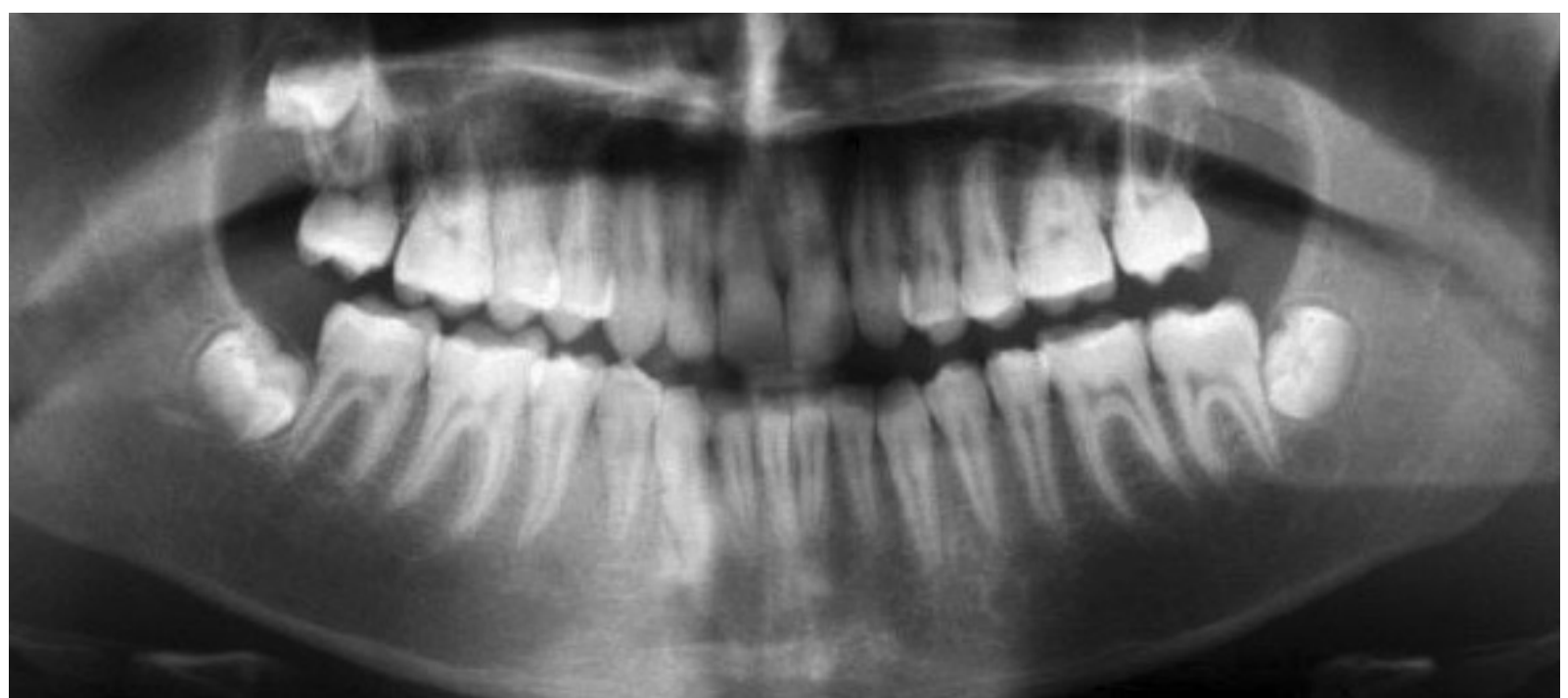

FIGURA 4B - Radiografia panorâmica do paciente MP, 14 anos na fase pós-tratamento.

\section{CONCLUSÃO}

A obtenção de excelentes resultados no tratamento ortodôntico de pacientes com agenesia de incisivo lateral superior é difícil, independente se a opção for abertura ou fechamento de espaços.
A questão fundamental nesses casos não é apenas a decisão de fechar ou abrir espaços, mas sim como atingir melhor resultado funcional e estético.

Enviado em: Outubro de 2002 Revisado e aceito: Julho de 2003 


\section{Treatment of Class II Division 1 with congenitally absent maxillary lateral incisor}

\section{Abstract}

This case report demonstrates the orthodontic treatment of a patient who presented a Class II Division 1 malocclusion, deep overbite and congenitally absent maxillary left lateral incisor. The space was closed orthodontically and maxillary cuspid was positioned in the place of the maxillary lateral incisor. The treatment applied resulted in a pleasant esthetics and normal occlusal relationship. The midline was coincident at the end of treatment and vertical discrepancy was properly corrected.

Key words: Missing lateral incisor. Class II Division1. Dental esthetics.

\section{REFERÊNCIAS}

1. $\mathrm{KOKICH} \mathrm{V.} \mathrm{G.} \mathrm{Managing} \mathrm{orthodontic-restorative} \mathrm{treatment} \mathrm{for}$ the adolescent patient. In: McNAMARA, J. A.; BRUDON W. L. Orthodontics and dentofacial orthopedics. Ann Arbor: Needham Press, 2001. p. 423-452.

2. McNEILL, R. W.; JOONDEPH, D. R. Congenitally absent maxillary lateral incisors: treatment planning considerations. Angle Orthod, Appleton, v. 43, p. 24-29, 1973.
3. SABRI, R. Management of missing maxillary lateral incisors. J Am Dental Assoc, Chicago, v. 130, p. 80-84, 1999.

4. STUART, C.; STALLARD, H. Diagnosis and treatment of occlusal relations of the teeth. Tex Dent J, Dallas v. 75, p. 430-435, 1957.

5. TUVERSON, D. L. Orthodontic treatment using canines in place of missing maxillary lateral incisors. Am J Orthod, St. Louis, v. 58, p. 109-127, 1970.
Endereço para correspondência

Roberto M. A. Lima Filho

Av. Alberto Andaló, 4025

S. J. Rio Preto - SP

CEP: $15015-000$

E-mail: robertolima@riopreto.com.br 